\title{
CORRELAÇÕES FENOTÍPICAS ENTRE CARACTERES VEGETATIVOS E DE PRODUÇÃO DE PALMITO DA PALMEIRA REAL AUSTRALIANA
} \author{
Saes $^{2}$ \\ ${ }^{1}$ Centro de Horticultura - IAC, C.P. 28 - CEP: 13001-970 - Campinas, SP. \\ ${ }^{2}$ Núcleo Experimental do Vale do Ribeira - IAC, C.P. 28 - CEP: 13001-970 - Campinas, SP. \\ ${ }^{3}$ Bolsista FAPESP. \\ *Autor correspondente <mlabovi@cec.iac.br>
}

Roberta Pierry Uzzo1,3; Marilene Leão Alves Bovi" ${ }^{1 *}$ Sandra Heiden Spiering1; Luiz Alberto

RESUMO: A seleção indireta é prática corrente no melhoramento de plantas perenes e vem sendo usada com sucesso em programas de melhoramento genético de palmeiras visando a produção de palmito. $O$ objetivo do trabalho foi auxiliar na identificação dos caracteres de natureza não destrutiva que possam ser utilizados futuramente na indicação de palmeiras aptas à colheita e na seleção indireta de plantas superiores de palmeira real australiana (Archontophoenix alexandrae Wendl. \& Drude). Em uma população de palmeiras com 30 meses de cultivo, foram estudados seis caracteres relacionados ao crescimento e seis caracteres componentes da produção de palmito através de análises de variabilidade, correlação e regressão múltipla. Entre os doze caracteres, o peso do coração, o peso do palmito, e palmito mais banda, mostraram maior variabilidade (CV acima de 53\%). Menor variabilidade foi encontrada para os caracteres diâmetro do palmito e comprimento da quarta folha (CV entre 26 e 22\%). O peso, o diâmetro e o comprimento do palmito mostraramse positivamente correlacionados com a altura e o diâmetro da planta. Entre os caracteres vegetativos não destrutíveis avaliados, a altura da planta foi o que mais contribuiu para a variação apresentada pelo peso total do palmito. Constitui assim um caráter útil, facilmente mensurável e de natureza não destrutiva que pode ser usado tanto para indicação de ponto ótimo de corte, quanto para a seleção de genótipos superiores dessa palmeira.

Palavras-chave: Archontophoenix, seleção indireta, melhoramento genético, caracteres agronômicos, genótipos superiores

\section{PHENOTYPIC CORRELATIONS BETWEEN VEGETATIVE CHARACTERS AND HEART-OF-PALM YIELD TRAITS OF THE AUSTRALIAN KING PALM}

\begin{abstract}
Indirect selection is a common practice in perennial crop breeding and has been frequently utilized for palms. Traits related to plant growth and heart-of-palm yield of the Australian king palm (Archontophoenix alexandrae Wendl. \& Drude) were studied in a 30 months old population, cultivated in Pariquera-Açu, SP, Brazil. The objective was to identify traits that can be subsequently used for the indication of plant prones to be harvested, as well as in the selection of superior palms. Correlation and multiple linear regression analyses were performed on the data. Among the twelve studied traits, the weights of edible basal stem, premium heart-of-palm, and heart-of-palm plus edible apical leaf presented the largest variability (CV above $53 \%$ ). Smaller variability was observed for heart-of-palm diameter and length of the fourth leaf (CV between 26 and $22 \%$ ). Highly positive correlation coefficients were found between heart-of-palm components (weight, diameter and length) and plant height and diameter. Among the vegetative traits, plant height contributed the most to the variation presented by the heart-of-palm total weight. It is a useful character, easily measurable and of non-destructive nature, that can be utilized to indicate the adequate timing for heart-of-palm harvesting, and for the selection of superior genotypes.

Key words: Archontophoenix, indirect selection, genetic improvement, agronomic traits, superior genotypes
\end{abstract}

\section{INTRODUÇÃO}

O palmito é conhecido e usado como alimento desde épocas remotas, sendo atualmente utilizado não só na culinária brasileira, como também na internacional. Denomina-se palmito o produto comestível, o qual é constituído por folhas ainda não desenvolvidas e imbricadas que saem a partir do meristema apical de palmeiras.
Várias palmeiras são usadas para a produção de palmito, sendo mais comuns aquelas do gênero Euterpe. No entanto, devido à extração desenfreada dessas, em pouco tempo a oferta será menor que a procura, já que o Brasil detém $95 \%$ do mercado de exportação mundial do palmito.

A palmeira real australiana (Archontophoenix spp.) vem ganhando a atenção de engenheiros agrônomos e produtores para a produção de palmito. 
Embora apenas o gênero Euterpe predomine ainda hoje nessa atividade, rapidamente cresce a participação de outros gêneros, quer seja em extração, ou cultivo tecnicamente realizado. Dessa forma, é expressiva atualmente a participação da pupunheira (Bactris gasipaes Kunth) no agronegócio palmito (Bovi, 2000), bem como tem aumentado o cultivo da palmeira real australiana para essa mesma atividade.

O gênero Archontophoenix é originário do leste da Austrália, sendo muito utilizado em praças e jardins brasileiros como planta ornamental. Pesquisas recentes mostraram que a palmeira real australiana possui um potencial grande para produção de palmito de qualidade e atestam a viabilidade de seu cultivo em nosso meio (Bovi, 1998). Seu palmito é do tipo nobre, com padrão de qualidade e sabor ainda superior ao das palmeiras do gênero Euterpe, embora também apresente rápido escurecimento após o corte. A colheita de palmito, nas espécies do gênero Archontophoenix, é feita em plantas com idade a partir de 30 meses de campo, desde que cultivadas em regiões aptas e com adubação apropriada (Bovi et al., 2001). As espécies tradicionais levam de 8 a 12 anos para estarem aptas para corte.

Poucos estudos são encontrados na área de genética e melhoramento em palmeiras, devido ao reduzido número de técnicos dedicados nessa área, bem como às dificuldades logísticas existentes por ser planta perene, de ciclo longo e grande altura.

Um dos parâmetros indispensáveis para o melhoramento de qualquer espécie é o conhecimento de correlações entre caracteres de interesse, por fornecer subsídios para a seleção ou descarte de materiais genéticos (Goldenberg, 1968; Simmonds, 1979). Estimativas de coeficientes de correlações fenotípicas simples, vêm sendo utilizadas com freqüência em plantas de ciclo longo, principalmente nas nativas, onde os experimentos nem sempre se encontram adequadamente delineados para estimar parâmetros genéticos (Bovi et al., 1990b). Em muitos casos, as correlações fenotípicas são consideradas suficientes para esclarecer relações entre caracteres de importância econômica dessas culturas (Bovi et al., 1992). Associações dessa natureza têm sido obtidas no melhoramento do palmiteiro e do açaizeiro para identificar caracteres vegetativos facilmente mensuráveis e não destrutíveis relacionados à produção de palmito (Bovi et al., 1990a; Bovi et al., 1991a; Bovi \& Godoy Jr., 1991; Fantini et al., 1997; Reis et al., 1999), bem como com a produção de frutos (Oliveira et al., 2000).

O presente trabalho teve por objetivo estimar as correlações fenotípicas entre caracteres relacionados ao crescimento e a produção de palmito, de forma a identificar aqueles mais facilmente mensuráveis, que possam ser, futuramente, utilizados na determinação de plantas aptas à colheita, bem como para a seleção daquelas fenotipicamente superiores a serem deixadas como plantas matrizes ou usadas em etapas posteriores no melhoramento genético da espécie.

\section{MATERIAL E MÉTODOS}

Medições envolvendo diferentes caracteres da planta e do palmito foram feitas dentro de uma população de palmeira real australiana, com aproximadamente 30 meses, em cultivo na Estação Experimental de Pariquera-Açu ( $24^{\circ} 43^{\prime} \mathrm{S}, 47^{\circ} 53^{\prime} \mathrm{W}$, e $25 \mathrm{~m}$ anm), SP. As sementes que deram origem a essa população foram fornecidas pela Epagri, Santa Catarina. O clima da região é mesotérmico, tropical, quente e úmido, com temperatura média anual de $21,8^{\circ} \mathrm{C}$, precipitação pluviométrica anual de $1587 \mathrm{~mm}$ e evapotranspiração de $1140 \mathrm{~mm}$.

O solo da área experimental, anteriormente classificado como Cambissolo Distrófico, de acordo com levantamento pedológico realizado por Sakai \& Lepsch (1984), é atualmente denominado Cambissolo Háplico distrófico. Trata-se de um solo moderadamente ácido, com teores de $\mathrm{Ca}$ e $\mathrm{K}$ baixo, mas com Mg relativamente alto, com drenagem moderada, relevo quase plano e saturação de bases variando de 20 a 40\% (Saes, 1995).

$\mathrm{O}$ espaçamento adotado na cultura foi de $2 \mathrm{x}$ $0,75 \mathrm{~m}$, onde se utilizaram mudas com seis de meses de idade, formadas em tubetes de $150 \mathrm{~mL}$ com substrato comercial. Durante o plantio foram colocados $100 \mathrm{~g}$ por cova de superfosfato simples. A partir do sexto mês de plantio iniciou-se a adubação mineral, empregando 50 g por planta da fórmula NPK 20:5:20, aplicados em cobertura e em faixas, a cada três meses.

Para o presente estudo foram cortadas, ao acaso, 238 plantas. Antes da colheita, os seguintes caracteres vegetativos foram medidos, de acordo com o estabelecido por Clement \& Bovi (2000): Diâmetro da planta - medido a 10 e a $50 \mathrm{~cm}$ acima da superfície do solo; Altura da planta - medida do solo até o ponto de inserção entre a flecha e a folha mais nova; Número de folhas - contado o número de folhas verdes completamente expandidas; Comprimento da ráquis foliar da quarta folha (folha +4) - medido do ponto de emissão dos folíolos até a bifurcação deles no ápice; Comprimento da flecha (folha 0) - medido do ponto de emissão na haste até o ápice da folha.

Os caracteres acima foram escolhidos pela facilidade de mensuração e, muitos deles, por terem apresentado boa correlação com caracteres do palmito em palmeiras de outros gêneros botânicos (Bovi et al., 1990b, 1993a, 1993b).

Após as mensurações, executou-se o corte da porção apical da palmeira (capitel) no local da amostragem, e as plantas, devidamente etiquetadas, foram levadas ao laboratório, onde se realizou a medição dos seguintes caracteres, também de acordo com o estabelecido por Clement \& Bovi (2000): Peso do coração, estipe tenro ou resíduo basal - porção do estipe, imediatamente abaixo do meristema, que é bastante tenro para ser consumido in natura; Diâmetro do palmito - diâmetro médio dos $\mathrm{n}$ toletes de palmito nobre (tipo 
exportação); Comprimento do palmito - número de toletes inteiros de palmito multiplicados por $9 \mathrm{~cm}$ (tamanho padrão do palmito tipo exportação); Peso do palmito exclusivamente o peso do palmito tipo exportação; Peso de banda ou folha tenra - peso da porção apical, ainda macia, mas não envolta por bainha foliar; Palmito + banda - peso do palmito propriamente dito (tipo exportação) mais a porção apical macia; Produto total somatório do palmito, banda e estipe macio.

Foi feita análise preliminar dos dados visando caracterizar a população amostrada para cada caráter estudado. Além da média, calculou-se desvio padrão, coeficiente e amplitude de variação e intervalo de confiança da média $(P=0,90)$, seguindo fórmulas descritas por Steel \& Torrie (1980). Dada a variabilidade existente (Bovi et al., 2001) e visando fornecer subsídios para trabalhos posteriores, foi calculado também o tamanho da amostra ideal, através do qual determinouse o tamanho da amostra que deveria ser tomada para que os dados estivessem variando em torno de $10 \%$ da média da população para os diversos caracteres estudados (Snedecor \& Cochran, 1974). As análises foram complementadas com o estudo de correlações lineares entre os diversos caracteres pelo método de Pearson, a $1 \%$ (Steel \& Torrie, 1980). Visto que os caracteres de natureza não destrutiva mostraram-se associados entre si, foi necessário considerar também as variações simultâneas entre eles. Desta forma, as análises foram complementadas por um estudo de regressão múltipla, assumindo-se o modelo linear e utilizando-se o processo de retirada das variáveis em passos, de acordo com o nível de significância do teste "t" a 1\% (Snedecor \& Cochran, 1974; Steel \& Torrie,
1980). Adotou-se como critério à retirada da variável cujo coeficiente de regressão foi estatisticamente igual a zero em nível de $1 \%$ pelo teste "t". Após a retirada dessa variável nova regressão era processada.

\section{RESULTADOS E DISCUSSÃO}

Embora as plantas tenham tido a mesma origem, foram cultivadas na mesma condição e sujeitas aos mesmos tratos culturais, houve variação muito grande, nas principais estimativas dos caracteres vegetativos $\mathrm{e}$ de produção dentro da população amostrada, sugerindo variabilidade genética (Tabela 1).

A população em estudo tinha, aos 30 meses após plantio no campo, diâmetro médio a $10 \mathrm{~cm}$ de altura do solo de $15,04 \mathrm{~cm}$, diâmetro médio a $50 \mathrm{~cm}$ de altura de $11,56 \mathrm{~cm}$, altura média de $148,72 \mathrm{~cm}$ e número médio de folhas fotossinteticamente ativas de 7,14. O comprimento da ráquis da quarta folha estava em torno de $166,72 \mathrm{~cm}$, enquanto que a folha flecha media em média $125,29 \mathrm{~cm}$.

A produção média de palmito foi de $198,50 \mathrm{~g}$, quando considerado apenas o tipo exportação, 247,51 g quando, além dele, foi incluído a porção apical macia e $541,29 \mathrm{~g}$ quando se considerou toda porção processável (palmito + banda + resíduo basal macio). O peso médio da porção macia do estipe (coração) foi $293,78 \mathrm{~g}, 48 \%$ superior a produção de palmito tipo exportação. O diâmetro médio do palmito esteve em torno de $3,24 \mathrm{~cm}$, enquanto que seu comprimento médio foi de $23,08 \mathrm{~cm}$.

Os valores máximo e mínimo tiveram muita variação para os caracteres destrutívos, sendo que o

Tabela 1 - Estimativas da média, intervalo de confiança, desvio padrão, coeficiente de variação, valores máximos e mínimos e tamanho da amostra ideal, para os caracteres estudados em uma população cultivada de Archontophoenix alexandrae. Tamanho da amostra $=238$. Núcleo de Agronomia do Vale do Ribeira, Pariquera-Açu, IAC, SP, 2000.

\begin{tabular}{|c|c|c|c|c|c|c|c|}
\hline Caracter & Média & $\begin{array}{l}\text { Intervalo de } \\
\text { confiança* }^{*}\end{array}$ & $\begin{array}{l}\text { Desvio } \\
\text { Padrão }\end{array}$ & $\begin{array}{l}\text { Coeficiente } \\
\text { de variação }\end{array}$ & Máximo & Mínimo & $\begin{array}{c}\text { Amostra } \\
\text { ideal }^{* *}\end{array}$ \\
\hline Diâme tro da planta a $10 \mathrm{~cm}(\mathrm{~cm})$ & 15,04 & $\pm 0,54$ & 4,22 & 28,08 & 24,63 & 4,66 & 30,29 \\
\hline Diâmetro da planta a $50 \mathrm{~cm}(\mathrm{~cm})$ & 11,56 & $\pm 0,46$ & 3,59 & 31,06 & 21,00 & 3,77 & 37,05 \\
\hline Altura da planta $(\mathrm{cm})$ & 148,72 & $\pm 7,23$ & 56,88 & 38,25 & 320,00 & 28,00 & 56,19 \\
\hline Número de folhas & 7,14 & $\pm 0,25$ & 1,97 & 27,60 & 13,00 & 1,00 & 29,26 \\
\hline Comprimento da quarta folha $(\mathrm{cm})$ & 166,72 & $\pm 4,73$ & 37,26 & 22,35 & 240,00 & 56,00 & 19,19 \\
\hline Comprimento da flecha $(\mathrm{cm})$ & 125,29 & $\pm 5,35$ & 42,13 & 33,62 & 230,00 & 10,00 & 43,42 \\
\hline Peso do coração $(\mathrm{g})$ & 293,78 & $\pm 20,03$ & 156,97 & 53,43 & 701,58 & 7,15 & 109,68 \\
\hline Diâmetro do palmito (cm) & 3,24 & $\pm 0,11$ & 0,82 & 25,40 & 5,80 & 0,80 & 24,78 \\
\hline Comprimento do palmito $(\mathrm{cm})$ & 23,08 & $\pm 1,10$ & 8,59 & 37,22 & 45,00 & 6,00 & 53,21 \\
\hline Peso do palmito $(\mathrm{g})$ & 198,50 & $\pm 15,93$ & 124,89 & 62,91 & 709,98 & 8,88 & 152,06 \\
\hline Peso da banda (g) & 49,01 & $\pm 17,14$ & 18,86 & 60,49 & 160,73 & 13,93 & 140,82 \\
\hline Palmito + banda $(g)$ & 247,51 & $\pm 18,34$ & 143,75 & 58,08 & 868,00 & 14,83 & 129,58 \\
\hline Produto total $(\mathrm{g})$ & 541,29 & $\pm 34,91$ & 273,63 & 50,55 & 1341,09 & 27,31 & 98,17 \\
\hline
\end{tabular}

*Intervalo de confiança com $P=0,05 ;{ }^{* *}$ Amostra ideal $\left[\left(t_{0,05} . \mathrm{CV}\right) / 10\right]^{2}$ 
peso do palmito teve valor máximo de 709,98 e mínimo $8,88 \mathrm{~g}$, o peso do palmito mais banda variou de 868,00 a $14,53 \mathrm{~g}$, o que conseqüentemente fez o valor do produto total variar muito.

Comparando-se principalmente os desvios padrão, os coeficientes de variação, os intervalos de confiança e os tamanhos da amostra ideal (Tabela 1), infere-se que os caracteres de produção são de avaliação muito menos precisa que os caracteres relacionados ao crescimento da planta. Resultados semelhantes foram obtidos quando de estudo similar em outras palmeiras (Bovi et al., 1990b).

Dentre os caracteres relacionados ao crescimento da planta, os mais variáveis foram a altura da planta e comprimento de flecha, com coeficientes de variação (CV) entre 33 a 39\%. Para esses caracteres, são necessários de 44 a 57 indivíduos para constituir uma amostra representativa da população.

Parece haver pouca variabilidade genética a ser explorada para a característica comprimento da ráquis foliar da quarta folha, pois, dentre os caracteres avaliados, esse foi o de menor variação (CV 22,35\%) e o de menor tamanho amostral para representar a população em estudo $(19,19$ plantas). As folhas das palmeiras crescem até um tamanho definido pela espécie (Tomlinson, 1990). No entanto, o mesmo autor esclareceu que o comprimento da ráquis foliar, bem como o número, o comprimento e a largura dos folíolos que compõem o limbo foliar são dependentes do estádio ontogenético da palmeira e limitados especialmente pelo potencial genético, pelas condições de sombreamento, bem como pela disponibilidade hídrica e nutricional. Clement (1995) encontrou para esse mesmo caráter, avaliado em pupunheira, coeficientes de variação entre 28 a $51 \%$, com diferenças nítidas entre populações. Pode-se aventar que a pouca variabilidade encontrada para o caráter comprimento da ráquis foliar, em relação aos demais caracteres avaliados e nas condições do experimento, seja devido aos seguintes fatores: palmeiras reais australianas da população estudada tenham base genética restrita; atingiram o comprimento máximo esperado para espécie já aos 30 meses de idade; e/ou que esse comprimento da ráquis tenha sido limitado por fatores hídricos e nutricionais.

$\mathrm{Na}$ Tabela 2, encontram-se os coeficientes de correlação linear simples entre os caracteres relacionados ao crescimento. Todos os caracteres estão correlacionados $(P<0,01)$, com a magnitude dos valores variando de 0,9399 a 0,4533. Menores magnitudes foram encontradas entre número de folhas e o comprimento da folha flecha $(r=0,4533)$ e entre o número de folhas e 0 comprimento da quarta folha $(r=0,5358)$. A maior magnitude $(r=0,9399)$ foi encontrada entre os diâmetros da planta medidos a 10 e a $50 \mathrm{~cm}$, como era de se esperar. Não obstante, maior significado para a seleção de plantas tem os valores encontrados entre diâmetros e altura $(0,8794$ a 0,8924$)$ e entre diâmetros e comprimento da quarta folha $(0,8252$ a 0,8420$)$.

Os caracteres de natureza destrutiva, que representam os componentes da produção (Tabela 3 ), também se mostraram alta e positivamente correlacionados entre si, com magnitudes variando de 0,9290 a 0,4043 , exceto para o par comprimento do palmito e peso da banda. Isso era esperado, visto que há uma relação aditiva entre o produto total e o peso de suas diferentes partes e uma relação multiplicativa entre diâmetro e comprimento, afetando diretamente o peso, tanto do produto total quanto do palmito tipo exportação (Clement, 1995; Clement \& Bovi, 2000). Coeficientes de correlação de elevada magnitude, referentes aos principais componentes da produção (especialmente àqueles relacionados ao peso, diâmetro e comprimento do palmito tipo exportação) foram obtidos anteriormente para o palmiteiro (Bovi et al., 1990a; Fantini et al., 1997), para a pupunheira (Bovi et al., 1992; Clement, 1995) e para o açaizeiro (Bovi et al., 1990b). No presente estudo, os maiores valores foram encontrados entre peso do palmito e peso do palmito + banda $(r=0,9290)$ e entre peso do coração e produção total $(r=0,9105)$. Dos pares em análise, apenas o comprimento do palmito com o peso de banda não mostrou coeficiente de correlação estatisticamente significativo $(r=0,1042)$. A falta de correlação significativa entre estes dois caracteres indica que não há uma associação linear, positiva ou negativa,

Tabela 2 - Estimativas dos coeficientes de correlação linear simples entre os caracteres relacionados ao crescimento da planta em uma população cultivada de Archontophoenix alexandrae. Tamanho da amostra $=238$. Núcleo de Agronomia do Vale do Ribeira, Pariquera-Açu, IAC, SP, 2000.

\begin{tabular}{|c|c|c|c|c|c|}
\hline Caracter & DPM & ALT & NFO & CQF & CFL \\
\hline Diâmetro da planta a $10 \mathrm{~cm}$ (DPB) & $0,9399^{* *}$ & $0,8924^{\star *}$ & $0,6424^{\star *}$ & $0,8420^{* *}$ & $0,6562^{* *}$ \\
\hline Diâmetro da planta a 50 cm (DPM) & & $0,8794^{\star \star}$ & $0,6779^{* *}$ & $0,8252^{\star *}$ & $0,6336^{* *}$ \\
\hline Altura da planta (ALT) & & & $0,6782^{* *}$ & $0,8409^{* *}$ & $0,6600^{* *}$ \\
\hline Número de folhas (NFO) & & & & $0,5358^{* *}$ & $0,4533^{* *}$ \\
\hline Comprimento da quarta folha (CQF) & & & & & 0,6270 ** \\
\hline Comprimento da flecha (CFL) & & & & & \\
\hline
\end{tabular}

${ }^{* *}$ Significativo a $P<0,01$. 
entre eles (Goldenberg, 1968; Steel \& Torrie, 1980). Dessa forma, o comprimento do palmito variou de forma independente à variação do peso da banda. No entanto, uma associação negativa entre esses caracteres era esperada, visto que quanto mais comprido o palmito, menor é a porção apical ainda aproveitável para processamento.

O caráter menos correlacionado com os componentes de produção foi o comprimento da folha flecha, sendo seguido pelo número de folhas (Tabela 4). Embora os coeficientes tenham sido significativos, a magnitude sempre foi comparativamente menor do que a dos demais. Dentre os componentes da produção, a magnitude foi menor nos pares que envolveram o comprimento do palmito. Mesmo em outras espécies de palmeiras esse componente sempre foi o de estimativa menos precisa (Bovi et al., 1990a, 1991b, 1992).

A variação nos caracteres facilmente mensuráveis, tais como altura, diâmetro e número de folhas vivas, indica que seleção fenotípica para esses caracteres na população estudada provavelmente trará progresso no programa de melhoramento dessa palmeira visando à produção de palmito. Embora o mesmo possa ser inferido com relação ao comprimento de flecha, ele é o que menos se correlacionou com a produção de palmito. Isso pode ser inferido mesmo com as estimativas de variabilidade e correlações sendo de natureza fenotípica. Quando os valores são altos e significativos e o controle local é satisfatório, há forte indicação de que os componentes genéticos também são altos. Para o comprimento da flecha, as mesmas considerações podem ser feitas, embora seja o que menos se correlacionou com a produção de palmito. Isto era de se esperar, pois a emissão da flecha é um processo fisiológico de expansão da folha e depende do estádio ontogenético das folhas internas, bem como do estado hídrico e nutricional das plantas (Tomlinson, 1990).

Um modelo completo de regressão múltipla linear foi estabelecido para cada caráter destrutivo, considerado como variável dependente, e as variáveis que não auxiliavam a explicação da variação observada foram retiradas, em passos, do modelo. Nesse caso, foi incluído nas equações apenas o diâmetro da planta a 10 $\mathrm{cm}$, pela maior precisão da medida em relação ao diâmetro a $50 \mathrm{~cm}$ (Tabela 5). As equações de regressão foram obtidas para as medidas expressas em centímetros e os pesos em gramas. A altura da planta foi a variável independente de maior contribuição para a estimativa da produção de palmito, tanto do tipo exportação, quanto do resíduo basal, do palmito mais banda e da produção total. Foi também a variável que mais contribuiu para a estimativa do comprimento do palmito. Resultado semelhante foi obtido anteriormente por Clement (1995) em pupunheira.

$\mathrm{Na}$ estimativa do diâmetro do palmito, a variável de maior contribuição foi o diâmetro da planta, aqui

Tabela 3 - Estimativas dos coeficientes de correlação linear simples entre os caracteres relacionados à produção de palmito, em uma população cultivada de Archontophoenix alexandrae. Núcleo de Agronomia do Vale do Ribeira, PariqueraAçu, IAC, SP, 2000.

\begin{tabular}{|c|c|c|c|c|c|c|}
\hline Caracteres & DPA & CPA & PP & PB & PEB & PDT \\
\hline Peso do coração (PC) & $0,6154^{* *}$ & $0,4043^{\star \star}$ & $0,5506^{* \star}$ & $0,5079^{* *}$ & $0,6367^{\star *}$ & $0,9105^{\star \star}$ \\
\hline Diâme tro do palmito (DPA) & & $0,4818^{* *}$ & $0,6874^{* *}$ & $0,5558^{* *}$ & $0,7478^{* *}$ & $0,7512^{* \star}$ \\
\hline Comprimento do palmito (CPA) & & & $0,7721^{* *}$ & 0,1042 & $0,5801^{* *}$ & $0,5412^{\text {** }}$ \\
\hline Peso do palmito (PP) & & & & $0,4902^{* *}$ & $0,9290^{* *}$ & $0,8115^{\star *}$ \\
\hline Peso da banda (PB) & & & & & $0,7208^{* *}$ & $0,6772^{* \star}$ \\
\hline Peso do palmito + banda (PEB) & & & & & & $0,8986^{\star \star}$ \\
\hline Produto total (PDT) & & & & & & \\
\hline
\end{tabular}

**Significativo a $P<0,01$.

Tabela 4 - Estimativas dos coeficientes de correlação linear simples entre os caracteres referentes ao crescimento da planta e à produção de palmito, em uma população cultivada de Archontophoenix alexandrae. Núcleo de Agronomia do Vale do Ribeira, Pariquera-Açu, IAC, SP, 2000.

\begin{tabular}{|c|c|c|c|c|c|c|c|}
\hline Caracter & $\mathrm{PC}$ & DPA & CPA & PP & PB & PEB & PDT \\
\hline Diâmetro da planta a $10 \mathrm{~cm}$ (DPB) & $0,6867^{* *}$ & $0,7332^{\star *}$ & $0,4476^{* *}$ & $0,6912^{\star *}$ & $0,6978^{* *}$ & $0,7882^{* *}$ & $0,8134^{* *}$ \\
\hline Diâmetro da planta a $50 \mathrm{~cm}$ (DPM) & $0,7339^{* *}$ & $0,7554^{\star *}$ & $0,4727^{\star *}$ & $0,7129^{* *}$ & $0,7098^{\star *}$ & $0,8132^{* *}$ & $0,8537^{* *}$ \\
\hline Altura da planta (ALT) & $0,7413^{* *}$ & $0,7008^{* *}$ & $0,5259^{* *}$ & $0,7164^{* *}$ & $0,6212^{* *}$ & $0,7869^{* *}$ & $0,8439^{* *}$ \\
\hline Número de folhas (NFO) & $0,5868^{* *}$ & $0,5567^{* *}$ & $0,3500^{* *}$ & $0,5342^{* *}$ & $0,5505^{\star *}$ & $0,6232^{* *}$ & $0,6681^{\star *}$ \\
\hline Comprimento da quarta folha (CQF) & $0,6758^{\star \star}$ & $0,6804^{\star *}$ & $0,4701^{* *}$ & $0,6236^{\star *}$ & $0,6035^{\star *}$ & $0,7008^{* *}$ & $0,7604^{\star *}$ \\
\hline Comprimento da flecha (CFL) & $0,5384^{* \star}$ & $0,4506^{* *}$ & $0,1853^{*}$ & $0,4241^{* *}$ & $0,5919^{* *}$ & $0,5636^{* *}$ & $0,6086^{* *}$ \\
\hline
\end{tabular}

**Significativo a $P<0,01,{ }^{*}$ a $P<0,05$. PC = peso do coração; DPA= diâmetro do palmito; CPA= comprimento do palmito; PP= peso do palmito; $\mathrm{PB}=$ peso da banda; $\mathrm{PEB}=$ peso do palmito + banda; $\mathrm{PDT}=$ produto total. 
Tabela 5 - Equações de regressão linear e múltipla e coeficientes de determinação, obtidos para os diferentes caracteres do palmito em relação aos caracteres de natureza não destrutiva da planta, em uma população cultivada de Archontophoenix alexandrae. Núcleo de Agronomia do Vale do Ribeira, Pariquera-Açu, IAC, SP, 2000.

\begin{tabular}{|c|c|c|}
\hline Variável dependente $(Y)$ & Equação de regressão linear, múltipla & $\begin{array}{c}\text { Coeficiente de } \\
\text { determinação }\left(R^{2}\right) \%\end{array}$ \\
\hline \multirow{3}{*}{ Peso do coração (PC) } & $\mathrm{PC}=-132,461+1,1278 \mathrm{ALT}+13,4411 \mathrm{NFO}+0,8317 \mathrm{CQF}$ & 56,26 \\
\hline & $P C=-57,0523+1,7550 A L T+12,3925 N F O$ & 55,33 \\
\hline & $P C=-11,6435+2,0451 \mathrm{ALT}$ & 54,20 \\
\hline \multirow{3}{*}{ Peso do palmito (PP) } & $P P=-69,4992+1,1374 A L T-0,5337 C F L+8,5953 D P B$ & 60,23 \\
\hline & $P P=-29,8623+1,1891 \mathrm{ALT}-0,4318 \mathrm{CFL}$ & 58,73 \\
\hline & $\mathrm{PP}=-52,1891+1,1679 \mathrm{ALT}$ & 57,71 \\
\hline \multirow{3}{*}{ Peso do palmito + banda (PEB) } & $\mathrm{PEB}=-170,8680+12,9431 \mathrm{DPB}+0,9702 \mathrm{ALT}+10,9232 \mathrm{NFO}$ & 71,36 \\
\hline & PEB $=-135,6850+13,8699 \mathrm{DPB}+1,1648 \mathrm{ALT}$ & 70,26 \\
\hline & $\mathrm{PEB}=-63,2202+2,0806 \mathrm{ALT}$ & 66,99 \\
\hline \multirow{3}{*}{ Peso da produção total (PDT) } & $\mathrm{PDT}=243,970+2,5109 \mathrm{ALT}+22,8161 \mathrm{NFO}+16,3655 \mathrm{DPB}$ & 75,62 \\
\hline & $\mathrm{PDT}=-167,220+3,5356 \mathrm{ALT}+25,2049 \mathrm{NFO}$ & 74,43 \\
\hline & $\mathrm{PDT}=-74,8637+4,1258 \mathrm{ALT}$ & 72,74 \\
\hline \multirow{3}{*}{ Diâme tro do palmito (DPA) } & $\mathrm{DPA}=0,6846+0,09314 \mathrm{DPB}+0,0620 \mathrm{NFO}+0,0042 \mathrm{CQF}$ & 54,67 \\
\hline & $\mathrm{DPA}=0,9267+0,1245 \mathrm{DPB}+0,0612 \mathrm{NFO}$ & 53,79 \\
\hline & $\mathrm{DPA}=1,0890+0,1427 \mathrm{DPB}$ & 53,72 \\
\hline \multirow{2}{*}{ Comprimento do palmito (CPA) } & $\mathrm{CPA}=14,5132+0,1095 \mathrm{AL}-0,0621 \mathrm{CFL}$ & 31,65 \\
\hline & $\mathrm{CPA}=11,2995+0,0789 \mathrm{ALT}$ & 26,71 \\
\hline
\end{tabular}

$\mathrm{ALT}=$ altura; $\mathrm{NFO}=$ número de folhas; $\mathrm{CQF}=$ comprimento da quarta folha; $\mathrm{CFL}=$ comprimento da flecha; $\mathrm{DPB}=$ diâmetro da planta a $10 \mathrm{~cm}$.

medido a $10 \mathrm{~cm}$ de altura do solo. O diâmetro do palmito está diretamente relacionado ao número de folhas internas do mesmo. Foi observado para a pupunheira que quanto maior o diâmetro da planta, maior é o número de folhas internas do palmito, portanto maior o seu diâmetro (Bovi et al., 1992). Depois da altura, essa foi a variável que maior contribuição forneceu às estimativas de produção, como pode ser observado pelo sinal e magnitude dos coeficientes de regressão múltipla presentes na Tabela 5. Dentre as variáveis em estudo, o diâmetro da planta só não foi importante na estimativa do peso do coração e do comprimento do palmito. Clement (1995) também não encontrou relação alométrica significativa entre essas variáveis em duas diferentes populações de pupunheira. Resultados semelhantes, referentes à importância principalmente da altura e do diâmetro da planta para a estimativa da produção futura de palmito foram obtidos para Euterpe edulis Mart. por outros autores e são atualmente a base do manejo sustentado para essa espécie (Fantini et al., 1997; Reis et al., 1999).

A contribuição do comprimento da quarta folha foi pequena, mesmo nas equações em que esse parâmetro estava presente (peso do coração e diâmetro do palmito). Semelhante foi à contribuição do comprimento de flecha. Nas equações em que aparece (peso do palmito e comprimento do palmito), a sua contribuição é negativa, ou seja quanto maior o comprimento da flecha, menor o peso e o comprimento do palmito.
O presente estudo evidencia que há variabilidade dentro de população não melhorada de palmeira real australiana. Mostra também a importância de alguns caracteres vegetativos, facilmente mensuráveis, ou mesmo visualmente estimados, para avaliação indireta da produção de palmito dessa palmeira. Dentre eles merecem destaque à altura e o diâmetro da planta. Por meio desses caracteres pode-se identificar plantas aptas à colheita, bem como selecionar aquelas fenotipicamente superiores que poderão ser deixadas como plantas matrizes ou usadas em etapas posteriores no melhoramento genético da espécie. No entanto, por se tratar de caracteres vegetativos, com forte componente ambiental, a seleção deve ser feita nas mesmas condições agrobioclimáticas.

\section{AGRADECIMENTOS}

Ao Carlos Augusto Colombo, Walter José Siqueira, Maria Eliza Ayres Guidetti Zagatto Paternianni e Charles Roland Clement pela revisão, críticas e sugestões.

À Epagri, Santa Catarina, pelo fornecimento das sementes que originaram a população base.

\section{REFERÊNCIAS BIBLIOGRÁFICAS}

BOVI, M.L.A.; GODOY JR., G. Juvenile-mature correlations in heart of palm plants. Revista Brasileira de Genética, v.14, p.739-751, 1991.

BOVI, M.L.A. Cultivo da palmeira real australiana visando à produção de palmito. Campinas: Instituto Agronômico, 1998, 26p. 
BOVI, M.L.A. O Agronegócio palmito de pupunha. O Agronômico, v.52, p.10$12,2000$.

BOVI, M.L.A.; GODOY JR., G.; SAES, L.A. Correlações fenotípicas entre caracteres da palmeira Euterpe edulis Mart. e produção de palmito. Revista Brasileira de Genética, v.14, p.105-121, 1991a.

BOVI, M.L.A.; GODOY JR., G.; CAMARGO, S.B.; SPIERING, S.H. Caracteres indiretos na seleção de pupunheiras inermes (Bactris gasipaes H.B.K.) para palmito. In: CONGRESO INTERNACIONAL SOBRE BIOLOGIA, AGRONOMIA E INDUSTRIALIZACION DEL PIJUAYO, 4., Iquitos, 1993. Anais. San José: UFCR, 1993a. p.163-176.

BOVI, M.L.A.; GODOY JR., G.; CAMARGO, S.B.; SPIERING, S.H. Seleção precoce em pupunheiras (Bactris gasipaes H.B.K.) para produção de palmito. In: CONGRESO INTERNACIONAL SOBRE BIOLOGIA, AGRONOMIA E INDUSTRIALIZACION DEL PIJUAYO, 4., Iquitos, 1993. Anais. San José: UFCR, 1993b. p.177-195

BOVI, M.L.A.; GODOY JR., G.; SPIERING, S.H.; CAMARGO, S.B. Correlações fenotípicas entre caracteres avaliados nos estádios juvenil e adulto de açaizeiros. Bragantia, v.50, p.321-334, 1991b.

BOVI, M.L.A.; GODOY JR., G.; SAES, L.A.; MORI, E.E.M. Subsídios para o sistema de manejo auto-sustentado do palmiteiro. Campinas: Instituto Agronômico, 1990a. 25p.

BOVI, M.L.A.; GODOY JR., G.; SPIERING, S.H.; CAMARGO, S.B. Relação entre caracteres da planta e do palmito de açaizeiros. Bragantia, v.49, p.69-81, 1990b.

BOVI, M.L.A.: SAES, L.A.; GODOY JR., G. Correlações fenotípicas entre caracteres não destrutíveis e palmito em pupunheiras. Revista Turrialba, v.42, p.382-390, 1992

BOVI, M.L.A.; SAES, L.A.; UZZO, R.P.; SPIERING, S.H. Adequate timing for heart-of-palm harvesting in king palm. Horticultura Brasileira, v.19, p.135139, 2001.

CLEMENT, C.R. Growth and genetic analysis of pejibaye (Bactris gasipaes Kunth, Palmae) in Hawaii. Honolulu, 1995. 95p. Dissertation (Ph.D.) University of Hawaii, Honolulu.

CLEMENT, C.R.; BOVI, M.L.A. Padronização de medidas de crescimento e produção em experimentos com pupunheira para palmito. Acta Amazonica, v.30, p.349-362, 2000.
FANTINI, A.C.; NODARI, R.O.; REIS, M.S.; REIS, A.; RIBEIRO, R.J. Estimativa da produtividade de palmito em plantas de palmiteiro (Euterpe edulis Martius) a partir de características fenotípicas. Revista Árvore, v.21, p.49-57, 1997.

GOLDENBERG, J.B. El empleo de la correlacion en el mejoramento genetico de las plantas. Fitotecnia Latinoamericana, v.5, p.1-8, 1968.

OLIVEIRA, M.S.P.; LEMOS, M.A.; SANTOS, V.F.; SANTOS, E.O. Correlações fenotípicas entre caracteres vegetativos e de produção de frutos em açaizeiros. Revista Brasileira de Fruticultura, v.22, p.1-5, 2000.

REIS, M.S.; CONTE, R.; FANTINI, A.C.; NODARI, R.O. Caracterização do incremento em diâmetro de Euterpe edulis Mart. e implicações para o seu manejo em formações florestais secundárias. Revista Árvore, v.23 p.413-422, 1999.

SAES, L.A. Resposta da bananeira "nanicão" à região do Vale do Ribeira. Piracicaba, 1995. 82p. Dissertação (Mestrado) - Escola Superior de Agricultura "Luiz de Queiroz", Universidade de São Paulo.

SAKAI, E.; LEPSCH, I.F. Levantamento pedológico detalhado da Estação Experimental de Pariqueraçu. Boletim Técnico do Instituto Agronômico n.83, p.1-56, 1984

SIMMONDS, N.W. Principles of crop improvement. New York: Longman, 1979. 408p.

SNEDECOR, G.W.; COCHRAN, W.G. Statistical methods. Ames: lowa State University Press, 1974. 543p.

STEEL, R.G.; TORRIE, J.H. Principles and procedures of statistics. New York: MacGraw-Hill, 1980.632p

TOMLINSON, P.B. The structural biology of palms. London: Clarendon Press Oxford, 1990. 463p.

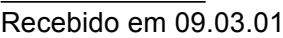

